\title{
Virality and the Virus: COVID-19 Cures on Twitter in India
}

\author{
Dibyendu Mishra \\ Microsoft Research, India \\ dibyendumishra96@gmail.com \\ Syeda Zainab Akbar \\ Microsoft Research, India \\ zainu9514@gmail.com
}

\author{
Gazal Shekhawat \\ Microsoft Research, India Microsoft Research, India \\ gazal.a.shekhawat@gmail.com joyojeet.pal@microsoft.com
}

\begin{abstract}
Social media platforms often become environments of information ambiguity during crisis events. We studied the discussion around four "cures" for COVID-19 in India, where the highest number of cases were recorded between 2020 and May 2021, focusing on the role played by high network accounts on social media such as those of journalists, politicians, and celebrities. We find that information scarcity and anxiety among citizens enabled non-experts, particularly the aforementioned social media influencers. We find that this undermined institutional sources of information and led to massive spikes in online interest around unproven cures during the peak of the crisis.
\end{abstract}

\section{Introduction}

India saw an unprecedented increase in the number of active COVID-19 cases in two waves. Notwithstanding several reports of undercounting, the first was in mid-2020 where India had a 7-day average of about 90,000 officially reported cases at its peak and, the second was in May 2021 where it had 7-day average of 380,000 officially reported cases at its peak[1]. The country's health resources were stretched, while shortages for oxygen, hospital beds, ICUs, testing, and mass cremations made for terrifying images on the streets and mainstream media. Added to this was political blame-shifting around lockdowns and deaths, leading to questions over institutional trust and widespread panic among citizens. With exponentially rising online interactions around public health at large, and the virus, in particular, messaging to help deal with the virus soon took the form of online information cascades.

The information disorder was accelerated during the second wave of the disease, starting in April 2021, when governmental communication receded, and the Prime Minister avoided press briefings through the worst period of the crisis. Meanwhile, platforms like Twitter were flooded with SOS calls, requests for hospital beds, oxygen etc. ${ }^{1}$. With the continuing lack of a dedicated COVID related drug, and uncertainty over access to vaccines at the time, the period saw much speculation around unproven cures and therapies. While these drugs are often put on off-label lists for compassionate usage, several studies outline the impacts of their cavalier usage in terms of shortages for other diseases [2] and the safety consequences[3].

The first phase of the crisis in India was marked by a loss of faith in institutions' ability to manage the crisis or communicate reliably[4], alongside an antagonistic information environment in which the state was seen as turning against its own[5]. In this paper we examine the information environment surrounding the crisis by focusing on the discussions around the "cures", specifically 3 off label cures i.e. Remdesivir, Hydroxychlorqouine and convalescent plasma therapy, and an alternative Ayurvedic drug i.e Coronil. We interpretively examine the tweets of key influential accounts around these drugs, the timing of their tweeting activity and their reach on social media through these tweets to characterise their impact.

\section{Crisis Informatics and the Twitterverse}

We situate our study under the literature of crisis informatics, which has for more than a decade, captured the evolving nature of emergency communications in light of new technologies. Crisis informatics was defined by Hagar[6] to refer to the linkages between institutions, individuals, and information and communication technologies that emerge during crises. Palen et al. added substantially to the concept and viewed emergency responses as an 'expanded social system' of information distribution between authorities and the general public[7]. Complementing a far longer history of disaster management studies, crisis informatics brings with it, present and future questions

\footnotetext{
${ }^{1}$ https://thewire.in/tech/what-the-sos-tweets-on-indian-twittertell-us-about-the-second-wave-of-covid-19
} 
of social behaviour and policy-making to aid a society transformed with the spread of the internet[7]. Social media sites, for instance, have emerged as prominent sources for information on public health[8]. Scholarship about this field has addressed emergent trends relating to natural calamities, shootings or extremist attacks, as well as public health emergencies.

Fischer notes that communication practices during crises are highlighted by people's needs to eliminate risks and reduce uncertainty[9]. Social media sites, in particular, offer a means to move away from the discomfort of the unknown, through the crowd-sourcing of known information, that leads to the emergence of mutual-aid or topical groups. The informational exchange, verification, and promotion on social media, can be seen as an exercise in expanding 'collective intelligence' about unfamiliar scenarios[7]. Here, networked users circumvent the conventional authority of official information releases, and are subsequently not bound by the same ethical and medico-legal concerns that drive centralised message production[7]. Several studies have documented successful online communications strategies. However, when it comes to public health, the persistence of false information, as well as a digital divide that skews their user-bases, platforms are far from being adequate replacements for traditional outbreak monitoring and prevention systems $[10,11]$.

Based on unique engagement metrics that come with social media platforms, a stream of research has also detailed the impact and presence of their most influential accounts. Influencers successfully reach out to a large audience by using both offline (areas of expertise or popularity) and online attributes (network centrality) $[12,13]$. Through a study of 1.6 million users studying 'diffusion events', Bakshy et al.[14] concluded that users who had already amassed a huge following were able to generate the largest information cascades. On Twitter, even though many accounts are infrequent or inactive, influencers solidify their positions due to the multiplicative interactions created by their followers through retweets or replies, that propels influencers on to newer feeds[15].

Holding three metrics to be particularly important on Twitter (number of followers, number of retweets received, and number of mentions) Cha et al.[16] used Spearman's rank correlation coefficient to find that highly followed users are not necessarily the ones who get high retweets or mentions on Twitter. However, by consistently engaging in a topic of their expertise top users gained disproportionately high engagement scores[16]. There are further variations in the usage of these metrics, for instance, tweet mentions might be used to address someone or notify them of a tweet[17]. Retweets have multiple meanings, they can be used to amplify someone else's message to newer audiences, to publicly agree with the source, to remind followers of their presence as listeners, or to gain followers through reciprocity[18].

Several studies expand on the emergence of subject matter influencers on Twitter. Based on findings of homophily in 'followership' networks on Twitter, Weng et al.[19] used machine learning to distill topics from tweets and combine them with a PageRank measure to rank influential users within each topic. One study finds[20] that topical experts share more trustworthy information and achieve remarkably high news and social media coverage doing so [35]. Overall, highly followed users receive high mentions and retweets across topics[16].

Due to the unprecedented scale of information that is presented to users of the internet, platforms and actors play a curatorial role in presenting this information[21]. Twitter influencers can also be considered as facilitators of this curation, as they project certain messages on followers' feeds and not others. Of the 7 types of curatorial roles Liu identifies[21], 'editors' who select or filter, storytellers who weave together a narrative, and docent members who teach or guide users are useful typologies of accounts during a public health crisis.

In the case of Twitter, users' amplification of emergency information varies significantly based on their proximity to the crisis[22, 23, 24]. For instance, directly impacted users retweet emergency-related content more often than those who are not[22]. Further, traditional media and official agencies enjoy greater popularity in the derivative information production[23, 25].

Previous research within crisis informatics that addresses public health concerns, provides a rich context for our study on COVID-19 related fallacies. Among health-related communication, the prominence of the anti-vaccine movement has been recurrent on social media even outside pandemic periods[26]. Studies on outbreaks of Ebola, Zika, and H1N1 reveal that information-seeking behaviours can fundamentally differ due to cultural connotations, hierarchy in communication models and political circumstances of affected regions[27, 10, 28]. This information-seeking is also particularly sensitive to gaps unaddressed by authorities. For instance, during the Zika outbreak in Latin America, despite being outside of the core infected regions, data from the US dominated knowledge networks worldwide. In light of such a situation, users devised a bottom-up practice of informational exchange, by seeking opinions from locals and recent travellers 
to particular regions via online forums[29]. While these approaches may have merits, they also come with problems of information validation or the amplification of anecdotes.

Recent work related to crisis informatics has focused on ICTs for crisis and emergency management[30], and disaster information and resilience, for emergency and crisis technologies[31]. Work in this domain has emphasized the changing information[32, 33] as well as relationships and ecologies[34, 35] over-time during disasters. For example, Wang et al.[35] state the case study of MERS-CoV epidemic in 2015 in South Korea to discuss the constantly changing response coordination plans according to the involvement of emerging actors with the previously predicted actors. Consistent with previous work in crisis informatics, such work has paid specific attention to using and classifying real-time data of disasters such as images and audio-logs[36, 37], effective use of large scale ICTs for supporting decision making[32, 33, 38], and employing user geotagging on twitter for enhancing Common Operational Picture Tools[39]. We find studies of Negre[40] and Siemon et al[41] in line with our findings which highlight the need for assurance of trust in digital and social networks. These studies focus on the issue of weakening credibility of experts on social networks and suggest newer models for enhancing trust. Work related to COVID-19 emergency responses underline the requirement for relationship management[34], behavioural change of systems[42, $43,44]$ and online conflict management $[45,46]$.

Limitations in existent crisis informatics research reflect those of social-media studies at large, as literature has mainly focused on the West[47]. Traditionally, studies have focused on crises that are specific to locations or sub-populations of concern, and subsequent data collection efforts have been guided by geographical anchoring[48]. However, there are increasing calls to bring together virtual data with offline messages to understand the dynamic nature of misinformation spread during crises[49]. However, the temporal longevity of the COVID-19 pandemic, its disproportionately large death toll across regions and age groups, makes it an exceptional emergency.

Thus, what is demanded of authorities, platforms and users can be extended and exponential, especially in countries where the pandemic has been prolonged amid healthcare and vaccine shortages. While previous crises have been geographically and temporally defined, the damage of COVID-19 also exposes the need for addressing the dysfunctions within crisis informatics. Idealistic models of information dissemination consider stakeholders as rational actors, however, as has been clear with COVID-19, authorities, public figures and civilians can play ambiguous roles in public health crises and mitigation may be secondary to politics, profit, or ideology.

\subsection{Information ambiguity: Misinformation, 'midinformation' and rumours}

Since the late 90 s, the study of misinformation and fake news has emerged as a separate field within its own right. The problem in tackling false information is a perennial one for platforms, with no simple solutions apparent, be it algorithmic modelling[50] or increased media-literacy[51]. The intentionality that has been commonly used to differentiate disinformation from misinformation, is also unclear in life and death situations induced by the healthcare shortages of the pandemic[50]. The evolving nature of scientific consensus when it comes to the effectiveness of disparate medications and therapies has also prompted the emergence of the term 'midinformation' ${ }^{2}$ to accommodate the ambiguity attached to the veracity of information shared even in good faith.

With an increase in the role of social networks in seeking and managing their care[52] and individuals replacing mainstream sources with social media as trusted sources of information[53, 54] the problem of misinformation and midinformation during the pandemic lead to detrimental consequences ${ }^{3}$. Additionally, the networked nature of Twitter does not allow messages to receive equal attention, and there is a 'giant effect' in rumour propagation since influential accounts with larger audiences can accelerate the snowballing of otherwise obscure information[55, 28]. 'Rumouring', which has parallels in misinformation spread, can be seen as a form of collective sense-making in situations of information vacuums from authorities in times of public anxieties[56]. Studies of this recurrent feature of crisis informatics also reveal that institutions, traditional media and influencers, can be instrumental in preventing or abetting the spread of rumours[56, 57]. A 2016 study of rumour propagation in an Australian hostage crisis notes several elements that resound with the communication failures during COVID-19[55]. Scenarios with high uncertainty, and a lack of official communication, can be complementary backdrops for rumours to emerge, and 'fill-in' information gaps[55]. The acceleration of how a message is forwarded is also resonant for Facebook, where public health information from accounts framed to be 'experts' can make information appear more credible to users[58].

\footnotetext{
${ }^{2}$ https://meedan.com/blog/missing-information-not-justmisinformation-is-part-of-the-problem/

${ }^{3}$ https://www.bbc.com/news/world-53755067
} 


\section{Data and Methods}

In this paper, we chose to study three separate categories of influencers - politicians, media entities, and other influencers(hereon referred to as just influencers). Here we define influencers as individuals in public life who enjoy heightened visibility as a result of their work in their respective occupational field, such as politics, journalism, sports, entertainment, business, academia, or their popularity may stem from a following-driven online presence - such as YouTube or Instagram influencers etc. We define media accounts as those belonging to individuals who identify as journalists, or to collectives such as news organizations. As we further discuss in section 4, we see these three categories as different stakeholders with their own motivations and roles in networks of information flow during crises. In the case of COVID-related output, we can assume that politicians' output is generally related to their party line, mainstream media journalists' output is generally related to their reporting and that of their organizations or allies, while other influencers such as entertainers, sportspersons, or businesspersons produce content related to their public relations, which may often be tied to the issues they wish to highlight within this frame. We use Twitter's Public API to collect tweets from these accounts from January 2020 to May 2021. Our methodology has two parts. We first discuss the curation of accounts for each category and then describe the retrieval of tweets relating to COVID-19 and medical drugs.

\subsection{Politician, Media and Influencer Datasets}

We use a publicly available data-set[59] of 37000 Indian politicians and their Twitter handles. Of these 14000 belong to the ruling party, the Bharatiya Janata Party(BJP), 12000 to the main opposition party, the Indian National Congress(INC), and the rest to various other national and regional parties in India. The set was curated using a machine learning classifier pipeline called NivaDuck and then hand-annotated by a group of human coders.

From January 2020, We collected an uninterrupted set of tweets from all of these politicians' accounts, 4100 media accounts (featuring journalists and media channels), and 2525 other influencers.

The list of journalists was hand-curated and supplemented with existing Twitter lists to create a seed set of about 100 verified journalists' accounts. We published this list and circulated them online, and pointed the list to various media professionals, to check if any major journalists were missing from our set and feel confident we were able to get a nearly complete set of at least the more influential journalists. We thereafter used the Twitter API to find the friends of each of the original set of accounts, giving us a set of over 50,000 Twitter accounts of those followed by the original 100. The assumption here is that journalists follow news-makers such as politicians and celebrities, as well as other journalists.

We first removed all the matches with politicians from the NivaDuck dataset. Then, a group of coders with contextual familiarity with Indian news matched accounts with known public figures. We used three parameters for classification. First, if the profile of the individual included words related to sports, entertainment, business, literature etc., and if they had a following greater than 20,000 , they were classified as influencers. If the account was blue-tick 'verified' we did no further check, else we manually verified through web searches. This method excludes people with influence who have a marginal presence on Twitter.

\subsection{Retrieval of tweets}

A bag of words approach was used to compile a set of tweets related to off-label and alternate drugs and therapies related to COVID-19. We focus on four highly discussed drugs and therapies and use them as keywords: 'hydroxychloroquine', 'plasma', 'remdesivir' and 'coronil'. We used these keywords to filter our corpus of lower-cased tweets. Since these terms are typically spelt out in English or used in hashtags, they returned tweets in various languages a majority of which were still in English. Using this method, we were able to retrieve 157100 tweets in total with 75396 tweets from politician handles, 56246 tweets from media, and 25458 tweets from influencers.

Next, we classify the tweets based on whether they were related to SOS calls/responses or not. We first build a seed set of high precision keywords related to SOS tweeting and increase the query size[60] by finding similar words in the rest of the dataset. We first filter the tweets set using the seed list of keywords and then train a Word2Vec[61] model on the resulting set. Using a cosine similarity measure, we then expand the seed set by finding the words with the highest pairwise similarity with the seed set. The final set of tweets classified as SOS tweets are the ones that contain either of the keywords from the expanded set.

\section{Stakeholders and Information Flows}

First, we mapped the stakeholders and flows of information in our data to draw a picture of the networks 
Table 1. Keywords and Hashtags for SOS tweets

\begin{tabular}{|c|l|}
\hline SOS & $\begin{array}{l}\text { \#covwords and Hashtags } \\
\text { arrange, donate, available, chahiye, help, } \\
\text { leads, need, require, request, urgent, helpline }\end{array}$ \\
\hline
\end{tabular}

and influences. The key stakeholders include (1) institutions that are involved in policy decisions, such as the Indian Council of Medical Research (ICMR), National Institute of Virology (NIV), World Health Organization (WHO) or government ministries and agencies, (2) hospitals and clinical professionals, (3) mainstream media, including journalists, media houses, fact-checkers etc., (4) politicians, (5) influencers, and finally, (6) the general public.

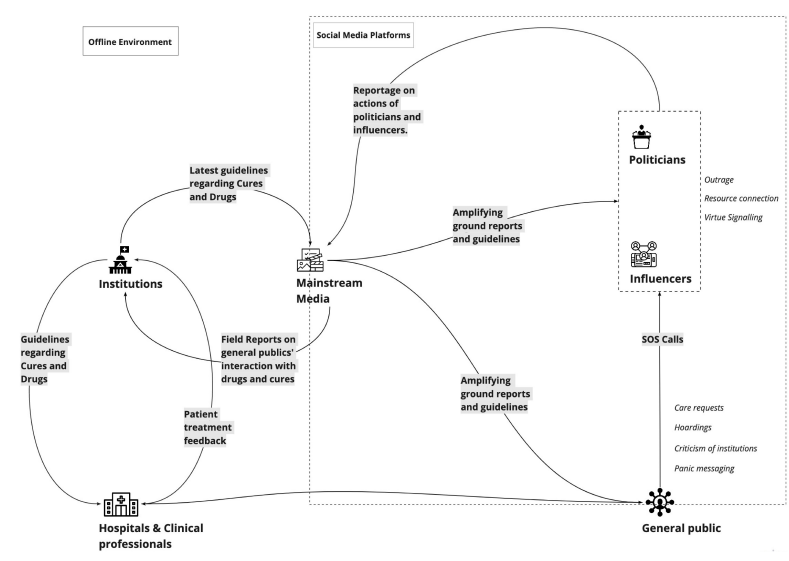

Figure 1. Flow of information on Twitter during events of crisis

In Figure1, we visualize the main flows of information observed in the data. Access to information is altered by affordances of social media platforms (filter bubbles, echo chambers, news-feed ranking)[62], therefore we can see that there is both an online and an offline realm in the information flows. We find that institutions are a key originator of information and that their information flows primarily outwards as directives and reports to the media and hospitals, but they also get information inwards in what the health systems locally and globally report back to them. The media act as the main nodes of information transmission to the general public, as well as to influencers and politicians, who emerge as key elements of the information environment. As we see in Table2, politicians had an even larger Twitter footprint on COVID-19 outreach on the four items studied than the media or influencers, suggesting that communicating with the electorate, and performing representative duties online was an important part of their social media engagement through the period.

We find that influencers and politicians played an instrumental role in amplifying ground reports, which were typically supplied by the general public. Thus, an alternate media ecosystem between citizens and those with influence to amplify messaging successfully ran in parallel to the official communication originating from institutions. These patterns support growing claims about a decline in the credibility of information by experts due to digital and social networks.[40] We find four broad themes of conversations that moved from the general public to politicians and influencers (measured through what they retweeted rather than the content they created themselves). These were mainly SOS calls (including care requests), hoarding reports, criticism of institutions, and panic messaging.

\section{Findings}

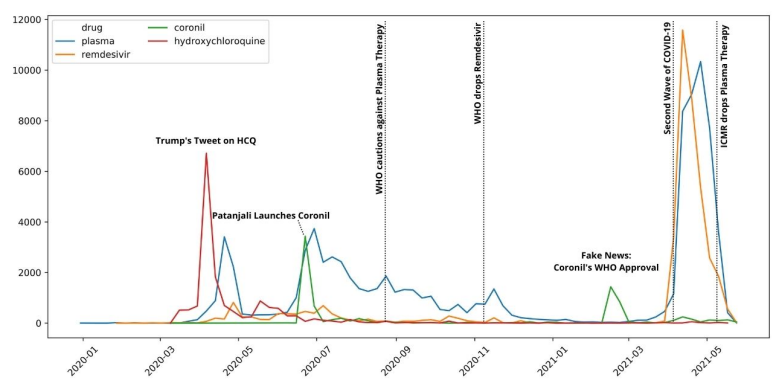

Figure 2. A timeline of weekly frequency of tweets for each drug/therapy from January 2020 to May 2021. The $y$ axis represents the total number of tweets corresponding to each drug in a given week.

In Figure2, we chart the conversation about the four drugs through the entire period of COVID-19 first and second waves. We see that while there is a large spike in engagement around April 2020, during the first wave of COVID-19 and the initial lockdown, the second wave in April 2021 has a much more precipitous rise in social media activity, especially around Plasma and Remdesivir. We see that the peaks are typically driven by a popular event in the news cycle, which may or may not be directly related to medical facts. For instance, Donald Trump's tweet threatening action against India for obstructing Hydroxychloroquine supplies was the driver of the first peak. Separately, the launch of Coronil by Patanjali led to the first peak on Coronil. While Hydroxychloroquine more or less disappeared from the timeline after its initial peak, we see that the second wave was a major driver for Remdesivir's popularity on Twitter. The drug witnessed more peaks than any 
other we studied and appears on the timeline more consistently than the rest.

More importantly, we see that updates in scientific medical information do not appear to immediately impact the action around a certain drug. For instance WHO warning against both Plasma and Remdesivir had no apparent impact on the spikes of their demand, much later in cycles of tweeting. In contrast, political controversies, celebrities weighing in on issues, all seem to correspond with spikes in engagement.

If we turn to Table2, we see that the overall extent of engagement (as measured through retweets from the influential accounts), was somewhat comparable across Plasma, Hydroxychloroquine and, Remdesivir, but Coronil has a much smaller footprint. However, we see that the total number of tweets for Plasma is much higher than for Remdesivir and Hydroxychloroquine. This is interesting because Plasma received the highest mentions as part of SOS messaging by far. Thus arguably, the therapy is the one that would typically need the most "retweeting" according to popular accounts. Whereas, the most political of all, Hydroxychloroquine, gets the highest engagement per tweet. We also see that influencers get the highest engagement per tweet on Hydroxychloroquine.

While the effectiveness of plasma therapy for Covid-19, was debunked in mid-2020, it continued to have SOS appeal, both in fits and starts through the latter half of 2020, but more significantly during the second wave in 2021. Indeed, we see in Table 2 varying degrees of the SOS messaging for each of the "cures" drive the discourse for each of the drugs. Coronil has very little SOS messaging since there was never really a shortage. The important thing though is that the spike of cases in the second wave threw most of the institutionally known data, such as that coming from the WHO or ICMR, out, and messaging was instead driven by panic, aided most by engagements of politicians and influencers.

We turn to an in-depth qualitative examination of the Twitter engagement around each of the four "cures". We did this by studying a timeline of the entire tweet thread of all messages, noting each message that got over 200 retweets, by studying the engagement of all major accounts $(100,000$ followers and above among politicians and influencers, 10,000 followers among journalists) with each to understand the engagement of influential voices, and through an interpretive examination of the first 100 and the most retweeted 100 messages for each case.

Remdesivir Remdesivir was one of the earliest discussed drugs by influencers, with the first tweet from a major account dating back to January 2020. Between
January and August 2020, there were a small number of engagements with Remdesivir, with occasional SOS calls and engagements around donations of Remdesivir by a major film star, Shah Rukh Khan. While this phase saw mentions of shortages, there was little frenzied engagement until April 11 2021, when there was a massive spike in tweets about Remdesivir. These were further driven by influencers like Dhruv Rathee offering to retweet and amplify messages about the need for Remdesivir by Twitter users. During this time we see a massive spike in tweets about the drug, which can be broken into three broad trends. First, were politicians and officials tweeting updates about the drug, as well as opposition politicians tweeting about healthcare failures. The second was journalists tweeting about shortages. And the third relates to journalists and influencers amplifying SOS messages about the drug. Some influencers actively solicited messages from the citizenry, offering to use their online following to bring attention to SOS situations. Starting around April 15 2021, journalists started posting helpline numbers, as well as the contact information of patients who needed drugs. Within days, celebrities started engaging with this messaging, flooding social media in the last two weeks of April. A film star, Sonu Sood, played a key role as an aggregator and amplifier of tweets. Sood had earlier come into into the media limelight during the first wave by providing transport services and advocating for displaced migrants. He came back to attention by offering to procure medical necessities such as drugs, beds for people who contacted him online and publicizing the cases where the drugs were delivered. Politicians were also active in this period, but they mainly argued back and forth, placing the blame for shortages either on the ruling party or the opposition.

Hydroxychloroquine The Twitter engagement around hydroxychloroquine was driven by the initial tweet from US President Donald Trump that suggested the drug was effective against COVID-19. Within a week, tweets reported shortages of hydroxychloroquine, a likely result of hoarding. Twitter engagement for the drug was overwhelmingly driven by political posturing rather than discussions around its effectiveness. After Donald Trump's public threat on Twitter against India for not releasing the drug, the Indian government revoked its ban on hydroxychloroquine exports, setting off a wave of activity online. On April 7, 2020, right after the government decided to export to the US, opposition leaders set off a flurry of messaging on Twitter. The establishment's to the opposition was also another social media PR blitz, starting with an announcement that Donald Trump had "followed" Modi 
Table 2. A summary of the number of tweets, total retweets and the percentage of SOS tweets of the drugs

\begin{tabular}{|l|l|l|l|l|l|l|l|l|l|}
\hline & \multicolumn{2}{|c|}{ Influencers } & \multicolumn{2}{c|}{ Politicians } & \multicolumn{2}{c|}{ Media } & \multicolumn{3}{c|}{ Total } \\
\cline { 2 - 10 } & Total & SOS & Total & SOS & Total & SOS & Total & SOS & Total Retweets \\
\hline Plasma & 10318 & $75.81 \%$ & 47351 & $73.36 \%$ & 28453 & $64.53 \%$ & 86122 & $70.74 \%$ & $20.86 \mathrm{M}$ \\
\hline HCQ & 1544 & $14.24 \%$ & 6674 & $16.73 \%$ & 7507 & $10.13 \%$ & 15725 & $13.34 \%$ & $28.77 \mathrm{M}$ \\
\hline Remdesivir & 3751 & $36.92 \%$ & 20270 & $34.41 \%$ & 17463 & $25.15 \%$ & 41484 & $30.74 \%$ & $29.43 \mathrm{M}$ \\
\hline Coronil & 1184 & $6.16 \%$ & 4006 & $5.71 \%$ & 3466 & $3.34 \%$ & 8656 & $4.82 \%$ & $3.96 \mathrm{M}$ \\
\hline Total & 16797 & $56.56 \%$ & 78301 & $54.99 \%$ & 56889 & $41.54 \%$ & 151987 & $50.13 \%$ & $83.04 \mathrm{M}$ \\
\hline
\end{tabular}

on Twitter, but not explicitly noting it as reciprocity. Many BJP politicians made potshots at the opposition leaders and asked for unity, and government-aligned journalists were brought into action. Right-wing journalist Aditya Raj Kaul emerged as the most influential voice online through the period. He engaged in largely pro-government tweeting about the benefits to foreign relations as an outcome of the government's giving away hydroxychloroquine to other countries. Thus a majority of social media activity we capture, was after the drug shortages had already set in.

Coronil Cornonil, saw highly divisive messaging, in part because it came to represent a culture war between believers in Indian traditional medicine and Western medicine. The drug is an Ayurvedic compound, marketed by Patanjali, one of India's largest producers of alternative medicine and fast moving consumer goods (FMCG). The main driver of the tweeting around the product was the owner of the company, Acharya Balakrishna, who has a massive following online. The drug had a blitz of news coverage starting on June 23, 2020, when tweets from mainstream news channels framed it as an evidence-based drug, some news channels even offering information about buying the drug. Politicians affiliated with the ruling party - Tajinder Bagga, Kapil Mishra and Priti Gandhi all tweeted about the drug in the last week of June, some proposing that a liberal conspiracy was holding back the country from accessing the compound's benefits. A rare SOS tweet related to Coronil came from Vikas Pandey of the ruling party IT cell, who spoke of a shortage but also advertised the drug, alongside two prominent right-leaning news anchors, Akhilesh Anand and Meenakshi Joshi. The most retweeted account on Coronil was that of a factchecker, who debunked stories about Coronil, followed by the account of a cartoonist, Satish Acharya, who lampooned the drug.

Plasma Of the four therapies studied here, plasma saw the most engagement by celebrities. Plasma got attention beginning with the first wave, with messages suggesting that plasma from recovered COVID-19 patient's blood could be used to treat the condition. At first, the engagements were driven in reaction to the othering of Muslims who were infected by COVID during the Tablighi Jamaat issue. In this round of social media engagements, Muslim survivors were presented as acting in the public interest, despite the attempts by politicians to present them as antagonists in the first wave. The social media engagement around plasma was further propelled by major politicians publicizing their own plasma donations starting in late April 2020. This narrative proved enduring, in late June, a major news network started a hashtag to get more plasma donations (\#donateplasmasavealife) and the convenors of the largest religious festival in Mumbai replaced celebrations with a plasma donation drive which went viral. There was very little said about plasma among politicians, influencers, and the media between October 2020 and April 2021. Till mid-May, Snapdeal, an Indian e-retailer, was running a plasma connection hashtag called \#Sanjeevani, despite its debunking a year ago.

\section{Discussion and Conclusion}

The "cures" studied here were largely debunked or not recommended by the international healthcare community, yet these institutional sources failed to provide clear answers, since the crisis was an evolving one, in turn laying the grounds for an information crisis[9]. What we see here is a unique intersection of influencer culture layered over an environment in which institutional knowledge was already being questioned. The WHO's mismanagement of information early in the crisis and its perceived geopolitical alignments, led to the distrust gaining political color, arguably making space for an often seen phenomenon - those with expanded social networks came to play an increasingly important role in information distribution[7]. In making sense of this case, we propose that the spread of cure misinformation is enabled by a heady intersection of digital vigilantism[57], and influencer culture[56] amplified in echo chambers[62] that were ripened in mistrust of government to turn to alternative sources to believe. 
The driver of social media action around Hydroxychloroquine was strikingly political and highlighted a form of antagonistic outrage messaging that sought to find targets of blame, rather than functional solutions to a crisis. Likewise, with Coronil, the lack of useful answers in official communication or hopeful data from medical science meant that people were amenable to buy into rumours of cure[55]. The systematic priming of citizens towards traditional and alternative remedies, especially from members of the ruling party and the government's Alternative Medicine (AYUSH) ministry set up the demand for Coronil online, alongside a large amount of messaging that debunked the compound.

While Hydroxychloroquine's mentions declined after the first wave, the persistence of both Plasma and Remdesevir as cures had terrible consequences. Clinicians were directly impacted, and reports from around the country came of doctors and hospitals being forced to prescribe Remdesevir, despite their knowledge of its ineffectiveness, since the families of patients held on to the possibilities they saw and demanded action in an otherwise hopeless situation.

The role of social media was further complicated the muddied information environment of April of 2021. As influencers with large followings actively triggered information cascades[14], social media quickly became a place to "perform" one's contribution. This was particularly dangerous in the case of SOS messaging. Thus, while journalists kept debunking these, influencers and politicians continued to talk about them, dominating social media attention for their performative concern.

As past work shows, those considered "local" to a disaster tend to less frequently share misinformation[24], but the nature of social media and the timeliness of SOS makes the 'locality' of individual senders of SOS messages less relevant as the terms of authentication are seconded to the reputation of senders. What we see here is that the amplification is not so much driven by proximity to a crisis[23], as it is to the performance of proximity to the crisis. Particularly in April 2021, during the peak of the healthcare system collapse, there was frenetic action.

Our work hopes to bring attention to a new turn for crisis informatics, that is not localized to one area, but that presents a direct risk or one-degree removed risk to virtually everyone who was engaged on social media through this period. Thus even if they were not directly ill or taking care of family, people were impacted by the feeling of fear at cases edging closer to their neighbourhoods, of healthcare system collapse that may impact general access to any kind of medical attention, or simple access to goods and services under the strict lockdown. In this crisis, for all practical purposes, everyone was involved. The situation thus elevates every influencer to a position of power, since all of their followers are arguably impacted. Consequently, we see here that the pandemic raises an entirely new set of concerns around influencer power and roles in a universal crisis.

The political economy of drugs in this situation needs to be closely interrogated. While the crisis around Coronil clearly emphasized tensions between mainstream and "traditional" medicine, there was also a nationalistic turn in the discourse around both Hydroxychloroquine and Remdesevir, or others where India is a producer[63]. The four drugs, and the action around them, resemble what Laclau has referred to as floating signifiers in democratic politics[64]. The "cures" became a site for for people to project their expectations of righteous policy-making, healthcare, and volunteerism. In the end, beyond any secondary motivations that come out in this work, what we are seeing in play is a massive crisis as it unfolds. The SOS calls, while amplified by influencers, were made by family members seeking any means to save their loved ones, running from pillar to post during a remarkable breakdown of the health system. While we can turn to what appears another step towards the weakening credibility of experts in digital and social networks [40, 41], what we are seeing here is a record of one of the greatest tragedies of our times, one in which verifiable information was one of the earliest casualties.

\section{References}

[1] H. Ritchie, E. Mathieu, L. Rodés-Guirao, C. Appel, C. Giattino, E. Ortiz-Ospina, J. Hasell, B. Macdonald, D. Beltekian, and M. Roser, "Coronavirus pandemic (covid-19)," Our World in Data, 2020. https://ourworldindata.org/coronavirus.

[2] J. D. Alpern and E. Gertner, "Off-label therapies for covid-19-are we all in this together?," Clinical Pharmacology \& Therapeutics, vol. 108, no. 2, pp. 182-184, 2020.

[3] N. Dasgupta, "Safety consequences of off-label drugs used for covid-19," Drug Safety, vol. 44, no. 4, pp. 399-402, 2021.

[4] T. Lancet, "India under covid-19 lockdown," Lancet (London, England), vol. 395, no. 10233, p. 1315, 2020.

[5] S. Z. Akbar, A. Panda, D. Kukreti, A. Meena, and J. Pal, "Misinformation as a window into prejudice: Covid-19 and the information environment in india," Proceedings of the ACM on Human-Computer Interaction, vol. 4, no. CSCW3, pp. 1-28, 2021.

[6] C. Hagar, "Using research to aid the design of a crisis information management course," in ALISE Annual Conference SIG Multicultural, Ethnic \& Humanistic Concerns (MEH). Information Seeking and Service 
Delivery for Communities in Disaster/Crisis, San Antonio, 2006.

[7] L. Palen, S. Vieweg, J. Sutton, S. B. Liu, and A. Hughes, "Crisis informatics: Studying crisis in a networked world," in Proceedings of the Third International Conference on E-Social Science, pp. 7-9, 2007.

[8] K. Vance, W. Howe, and R. P. Dellavalle, "Social internet sites as a source of public health information," Dermatologic clinics, vol. 27, no. 2, pp. 133-136, 2009.

[9] D. Fischer, "Social networking sites in the aftermath of a crisis-the enabling role for self-organization," in Proceedings of the 51st Hawaii International Conference on System Sciences, 2018.

[10] M. Abouzahra and J. Tan, "Twitter vs. zika-the role of social media in epidemic outbreaks surveillance," Health Policy and Technology, vol. 10, no. 1, pp. 174-181, 2021.

[11] D. Chernobrov, "Digital volunteer networks and humanitarian crisis reporting," Digital Journalism, vol. 6, no. 7, pp. 928-944, 2018.

[12] J. Goldenberg, S. Han, D. R. Lehmann, and J. W. Hong, "The role of hubs in the adoption process," Journal of marketing, vol. 73, no. 2, pp. 1-13, 2009.

[13] D. Kempe, J. Kleinberg, and É. Tardos, "Maximizing the spread of influence through a social network," in Proceedings of the ninth ACM SIGKDD international conference on Knowledge discovery and data mining, pp. 137-146, 2003.

[14] E. Bakshy, J. M. Hofman, W. A. Mason, and D. J. Watts, "Everyone's an influencer: quantifying influence on twitter," in Proceedings of the fourth ACM international conference on Web search and data mining, pp. 65-74, 2011.

[15] I. Anger and C. Kittl, "Measuring influence on twitter," in Proceedings of the 11th international conference on knowledge management and knowledge technologies, pp. 1-4, 2011.

[16] M. Cha, H. Haddadi, F. Benevenuto, and K. Gummadi, "Measuring user influence in twitter: The million follower fallacy," in Proceedings of the International AAAI Conference on Web and Social Media, vol. 4, 2010.

[17] C. Honey and S. C. Herring, "Beyond microblogging: Conversation and collaboration via twitter," in $200942 n d$ Hawaii International Conference on System Sciences, pp. 1-10, Ieee, 2009.

[18] d. boyd, S. Golder, and G. Lotan, "Tweet, tweet, retweet: Conversational aspects of retweeting on twitter. hicss-43," IEEE: Kauai, HI, vol. 6, 2010.

[19] J. Weng, E.-P. Lim, J. Jiang, and Q. He, "Twitterrank: finding topic-sensitive influential twitterers," in Proceedings of the third ACM international conference on Web search and data mining, pp. 261-270, 2010.

[20] M. B. Zafar, P. Bhattacharya, N. Ganguly, S. Ghosh, and K. P. Gummadi, "On the wisdom of experts vs. crowds: Discovering trustworthy topical news in microblogs," in Proceedings of the 19th ACM Conference on Computer-Supported Cooperative Work \& Social Computing, pp. 438-451, 2016.

[21] S. B. Liu, "The rise of curated crisis content," in Proceedings of the Information Systems for Crisis Response and Management Conference (ISCRAM 2010), 2010.
[22] K. Starbird and L. Palen, Pass it on?: Retweeting in mass emergency. International Community on Information Systems for Crisis Response and ..., 2010.

[23] K. Starbird, L. Palen, A. L. Hughes, and S. Vieweg, "Chatter on the red: what hazards threat reveals about the social life of microblogged information," in Proceedings of the 2010 ACM conference on Computer supported cooperative work, pp. 241-250, 2010.

[24] R. Thomson and N. Ito, "Social responsibility and sharing behaviors online: the twitter-sphere's response to the fukushima disaster," International Journal of Cyber Society and Education, vol. 5, no. 1, pp. 55-74, 2012.

[25] C. Chew and G. Eysenbach, "Pandemics in the age of twitter: content analysis of tweets during the $2009 \mathrm{~h} 1 \mathrm{n} 1$ outbreak," PloS one, vol. 5, no. 11, p. e14118, 2010.

[26] A. M. Jamison, D. A. Broniatowski, M. Dredze, Z. Wood-Doughty, D. Khan, and S. C. Quinn, "Vaccine-related advertising in the facebook ad archive," Vaccine, vol. 38, no. 3, pp. 512-520, 2020.

[27] H. Ding and J. Zhang, "Social media and participatory risk communication during the h1n1 flu epidemic: A comparative study of the united states and china," China Media Research, vol. 6, no. 4, pp. 80-91, 2010.

[28] T. K. Sell, D. Hosangadi, and M. Trotochaud, "Misinformation and the us ebola communication crisis: analyzing the veracity and content of social media messages related to a fear-inducing infectious disease outbreak," BMC Public Health, vol. 20, pp. 1-10, 2020.

[29] X. Gui, Y. Kou, K. H. Pine, and Y. Chen, "Managing uncertainty: using social media for risk assessment during a public health crisis," in Proceedings of the 2017 CHI conference on human factors in computing systems, pp. 4520-4533, 2017.

[30] M. Turoff, J. Dugdale, and E. Negre, "Introduction to the minitrack on information and communication technologies for crisis and emergency management," in Proceedings of the 53rd Hawaii International Conference on System Sciences, 2020.

[31] E. Negre, F. Benaben, M. Sakurai, J. Dugdale, and A. Tapia, "Introduction to the minitrack on disaster information, resilience, for emergency and crisis technologies," in Proceedings of the 54th Hawaii International Conference on System Sciences, p. 2203, 2021.

[32] K. Meesters and Y. Wang, "Information management in large-scale disaster exercises: An integrated perspective," 2020.

[33] J. Coche, G. R. Rodriguez, A. Montarnal, A. Tapia, and F. Benaben, "Social media processing in crisis response: an attempt to shift from data to information exploitation.," in HICSS 2021-54th Hawaii International Conference on System Sciences, pp. 2285-2294, 2021.

[34] D. Pamukcu and C. Zobel, "Characterizing 311 system reactions to a global health emergency," in Proceedings of the 54th Hawaii International Conference on System Sciences, p. 2216, 2021.

[35] C. Wang, Y. Kim, and S. S. Oh, "Epidemic response coordination networks in "living documents"," 2020.

[36] M. Johnson, D. Murthy, B. Roberstson, R. Smith, and K. Stephens, "Disasternet: Evaluating the performance of transfer learning to classify hurricane-related images posted on twitter," in Proceedings of the 53rd Hawaii International Conference on System Sciences, 2020. 
[37] K. Steen-Tveit, J. Radianti, and B. E. Munkvold, "Using audio-logs for analyzing the development of a common operational picture in multi-agency emergency response," in Proceedings of the 53rd Hawaii International Conference on System Sciences, 2020.

[38] M. Zhao and R. John, "Building community resilience using gain-loss framing to nudge homeowner mitigation and insurance decision-making," in Proceedings of the 54th Hawaii International Conference on System Sciences, p. 2206, 2021.

[39] N. Elrod, H. Hall, P. Mahajan, R. Grace, and J. Kropczynski, "Data census of a geographically-bounded tweet set to enhance common operational picture tools," in Proceedings of the 54th Hawaii International Conference on System Sciences, p. 2245, 2021.

[40] E. Negre, "Crisis management and distrust: Study of an industrial accident in france," in Proceedings of the 54th Hawaii International Conference on System Sciences, p. $2235,2021$.

[41] C. Siemon, D. Rueckel, and B. Krumay, "Blockchain technology for emergency response," in Proceedings of the 53rd Hawaii International Conference on System Sciences, 2020.

[42] M. Zetterholm, P. Elm, and S. Salavati, "Designing for pandemics: a design concept based on technology mediated nudging for health behavior change," in 54th Hawaii International Conference on System Sciences, January 4-8, 2021, pp. 3474-3483, University of Hawai' i, 2021

[43] P. Vogel, C. Kurtz, C. Grotherr, and T. Böhmann, "Fostering social resilience via online neighborhood social networks during the covid-19 pandemic and beyond: Status quo, design dilemmas and research opportunities," in Proceedings of the 54th Hawail International Conference on System Sciences, p. 3037, 2021.

[44] C. Chan, D. Qiu, and F. Tan, "Smart community and social resilience: Reflection on the covid-19 pandemic," in Proceedings of the 54th Hawaii International Conference on System Sciences, p. 2444, 2021.

[45] F. De Franca, C. Dos Santos, C. Penteado, D. Goya, L. Mazim, C. Kamienski, D. Di Genova, and D. Ramos, "Dynamics of conflicts on the twitter social network: a case study on the use of chloroquine in brazil," in Proceedings of the 54th Hawaii International Conference on System Sciences, p. 2976, 2021.

[46] C. Kamienski, L. Mazim, C. Penteado, D. Goya, D. Di Genova, F. De Franca, D. Ramos, and F. Horita, "A polarization approach for understanding online conflicts in times of pandemic: A brazilian case study," in Proceedings of the 54th Hawaii International Conference on System Sciences, p. 2101, 2021.

[47] C. Reuter, A. L. Hughes, and M.-A. Kaufhold, "Social media in crisis management: An evaluation and analysis of crisis informatics research," International Journal of Human-Computer Interaction, vol. 34, no. 4 , pp. 280-294, 2018.

[48] C. Hagar, "Introduction to the special section," Bulletin of the American Society for Information Science and Technology, vol. 36, no. 5, pp. 10-12, 2010.

[49] P. Pond, "The space between us: Twitter and crisis communication," International Journal of Disaster Resilience in the Built Environment, 2016.
[50] S. Kumar and N. Shah, "False information on web and social media: A survey," arXiv preprint arXiv:1804.08559, 2018.

[51] S. Badrinathan, "Educative interventions to combat misinformation: Evidence from a field experiment in india," APSA Preprints, 2020.

[52] C. O'Connor and J. O. Weatherall, The misinformation age: How false beliefs spread. Yale University Press, 2019.

[53] E. Zuckerman, "Mistrust, efficacy and the new civics: Understanding the deep roots of the crisis of faith in journalism," 2017.

[54] N. Newman and R. Fletcher, "Bias, bullshit and lies: Audience perspectives on low trust in the media," Available at SSRN 3173579, 2017.

[55] A. Arif, K. Shanahan, F.-J. Chou, Y. Dosouto, K. Starbird, and E. S. Spiro, "How information snowballs: Exploring the role of exposure in online rumor propagation," in Proceedings of the 19th ACM Conference on Computer-Supported Cooperative Work \& Social Computing, pp. 466-477, 2016.

[56] C. Andrews, E. Fichet, Y. Ding, E. S. Spiro, and K. Starbird, "Keeping up with the tweet-dashians: The impact of'official'accounts on online rumoring," in Proceedings of the 19th ACM Conference on Computer-Supported Cooperative Work \& Social Computing, pp. 452-465, 2016.

[57] K. Starbird, J. Maddock, M. Orand, P. Achterman, and R. M. Mason, "Rumors, false flags, and digital vigilantes: Misinformation on twitter after the 2013 boston marathon bombing," IConference 2014 Proceedings, 2014.

[58] P. Borah and X. Xiao, "The importance of 'likes': The interplay of message framing, source, and social endorsement on credibility perceptions of health information on facebook," Journal of health communication, vol. 23, no. 4, pp. 399-411, 2018.

[59] A. Panda, A. Gonawela, S. Acharyya, D. Mishra, M. Mohapatra, R. Chandrasekaran, and J. Pal, "Nivaduck - a scalable pipeline to build a database of political twitter handles for india and the united states," in International Conference on Social Media and Society, SMSociety'20, (New York, NY, USA), p. 200-209, Association for Computing Machinery, 2020.

[60] P. Vijayaraghavan, S. Vosoughi, and D. Roy, "Automatic detection and categorization of election-related tweets," in Proceedings of the International AAAI Conference on Web and Social Media, vol. 10, 2016.

[61] T. Mikolov, K. Chen, G. Corrado, and J. Dean, "Efficient estimation of word representations in vector space," arXiv preprint arXiv:1301.3781, 2013.

[62] S. Flaxman, S. Goel, and J. M. Rao, "Filter bubbles, echo chambers, and online news consumption," Public opinion quarterly, vol. 80, no. S1, pp. 298-320, 2016.

[63] C. Adolph, K. Amano, B. Bang-Jensen, N. Fullman, and J. Wilkerson, "Pandemic politics: Timing state-level social distancing responses to covid-19," Journal of Health Politics, Policy and Law, vol. 46, no. 2, pp. 211-233, 2021.

[64] E. Laclau, "Populism: What's in a name?," Populism and the Mirror of Democracy, vol. 48, 2005. 